\title{
Power-Controlled Data Transmission in Wireless Ad- Hoc Networks: Challenges and Solutions
}

\author{
Bilgehan Berberoglu \\ Department of Computer Engineering \\ Fatih University \\ Istanbul, Turkey
}

\author{
Taner Cevik \\ Department of Computer Engineering \\ Fatih University \\ Istanbul, Turkey
}

\begin{abstract}
Energy scarcity and interference are two important factors determining the performance of wireless ad-hoc networks that should be considered in depth. A promising method of achieving energy conservation is the transmission power control. Transmission power control also contributes to the mitigation of interference thereby promotes throughput by means of rendering multiple hosts to communicate in the same neighborhood simultaneously without impairing each other's transmissions. However, as identified previously in the literature, traditional hidden terminal problem gets deteriorated when transmission power control mechanism is intended to be applied. In this article, we discuss the primary details about the power usage and throughput deficiency of the traditional 802.11 RTS/CTS mechanism. Improvements by means of power control are introduced as well as the solutions to the challenges likely to emerge because of the usage of diverse power levels throughout the network.
\end{abstract}

Keywords-ad-hoc networks; energy conservation; power control; throughput

\section{INTRODUCTION}

Wireless ad-hoc networks (WANETs) have gained increasing popularity because of the ubiquitous communication needs with satisfying costs. Data communication is irrevocable for many people and applications in fields such as military, medical and industry [1]. Moreover, the easy, low cost deployment and set-up of WANETs have played an important role in the proliferation of that technology in fields such as hotels, airports, fabricates, surveillance areas, etc. [2]. A WANET consists of mobile wireless devices that can start communication immediately when get together in a reasonable proximity which is also called the transmission distance. In doing so, the lack of need for a centralized coordinator to administrate the communication and also an infrastructure for data transmission are the most prominent features of WANETs [3] that made them a globally prevalent technology especially for the last mile. Furthermore, instead of the need of an direct communication establishment to a central coordinator (no connection unless the distance is inside the transmission range) in order to connect to the internet, hosts can maintain data transmission by means of relaying each other's data through the gateway point which is called multi-hop communication. Besides, any group of nodes in the network can start communication with each other without redundantly sending their data to an access point [4].

Though it is indisputable that WANETs facilitate many processes in a variety of application fields, they unfortunately embody serious characteristic challenges and problems to be concerned with. The two important challenges of wireless communications is the bandwidth scarcity and limited energy supplies of the mobile devices used. Since mobile devices are battery enabled and the communication unit drains a significant portion [5-6], it is intended to develop highly energy-efficient protocols and architectures to be utilized in WANETs. Those researches that are focused on energy and bandwidth efficiency mainly developed for the second layer of the protocol stack that is Medium Access Control (MAC). MAC layer is of particular importance for WANETs because it coordinates the multiple accesses to the common transmission medium among hosts [7], and also maintains the reliability of the transmissions.

MAC layer contributes to the energy conservation in a way by coordinating also the transmission power levels of the hosts thereby preventing the redundant energy consumption because of the data transmission at maximum power level. That is, instead of a node to transmit its data to the targeted host with a constant high power unnecessarily, it is possible to use a reasonable lower power level to achieve a desired data communication which is called power controlled data transmission (PCDT). By employing PCDT mechanism, hosts can arrange their power levels according to the distance to the next hop of the packets dynamically. Since it is possible to define different routes for a stream of data packets, next hops that will relay them through the ultimate receiver may also change. That is, conveying data to a closer point requires lower energy consumption.

Utilizing dynamic power levels dynamically by means of PCDT also exploits interference reduction. Since lower energy signals do not propagate to further distances, the size of the area they affect in terms of interference also reduces. By this way, more hosts possibly stay out of the interfering range of the node and can start communication without being affected by the ongoing transmission which consequently results with throughput improvement in the network.

One of the primary challenges in wireless communications to be considered is the hidden terminal problem that is firstly identified by Tobagi and Kleinrock [8-9]. Since it is not possible for a sender to detect a collision at the receiver side, hosts lay at the opposite side of the receiver are hidden to the sender. Thus, the sender will not be able to recognize an ongoing receive process at the receiver and might start to transmission which will result as a collision at the receiver. The fundamental solution offered to remedy the hidden-node 
problem is the Multiple Access with Collision Avoidance (MACA) [10] that utilizes a handshake mechanism by exchanging signaling messages called Request-to-Send (RTS) / Clear-To-Send (CTS). Taking reference the MACA method, many research activities have been performed to achieve improvements and amendments.

Unfortunately, PCDT resurrects the hidden-terminal problem which has been obviated previously, because using a dynamic power level lower than a definite one leads to an expansion of the interference range at the receiver side which turns out new hidden terminal candidates to emerge that previously not. Many research activities have been performed in order to mitigate this newly emerged hidden-terminal challenge induced by the power control mechanism. In this article, we discuss the primary details about the power usage and throughput deficiency of the traditional 802.11 RTS/CTS mechanism. Improvements by means of power control are introduced as well as the solutions to the challenges likely to emerge because of the usage of diverse power levels throughout the network.

The rest of this paper is organized as follows. Section II gives details about the fundamental concepts such as traditional hidden and exposed terminal problems, spatial reusing as well as some of the solutions offered so far in the literature. Section III introduces power controlled transmission challenges and solutions. Finally, Section IV concludes the paper and provides an outline of future directions.

\section{PRELIMINARIES}

One of the most important layers of the communication protocol stack is the portion of the second layer that is called MAC. As is known, MAC layer coordinates the access to the common transmission medium as fair as possible among the sharing hosts. IEEE 802.11 standard [11] is the de facto standard preferred for local area communications. MAC protocols are roughly classified into two categories as: deterministic schedule-based protocols and stochastic contention-based protocols [12].

Schedule-based protocols such as 802.11 Point Coordination Function (PCF) depends on strict time synchronization; which is difficult to maintain in terms of resource consumption that is very critical for some types of networks such as Wireless Sensor Networks (WSNs). Each node in the network is assigned a dedicated slot permanently or dynamically depending on the type and Quality-of-Service (QoS) requirements of the application or network. However, once this complex synchronization challenge is handled successfully, guarantees for the demands such as delay, bandwidth, etc. can be achieved easily. In contrast, contentionbased protocols such as IEEE 802.11 distributed Coordination Function (DCF) do not give guarantees like delay or bandwidth. There is not any central coordinator in the network that allocates resources among the hosts. Every node senses and competes for the common transmission medium and starts transmission unless it detects any signal that is already on the way. Otherwise, it refrains itself from transmission and makes attempt later some time. The lack of the need for the synchronization of the hosts and presence of a central coordinator makes contention-based protocols relatively easier to impose [13-14]. Thus, substantial portion of the efforts have been devoted to the development of new ideas or improvement of the former contention-based protocols.

As mentioned in the previous section, the fundamental approach applied in contention-based protocols is the RTS/CTS mechanism. The RTS/CTS mechanism remedies one of the most crucial challenges in wireless communication that is hidden terminal problem. Details about the hidden terminal problem and RTS/CTS mechanism are introduced in the following section.

\section{A. Hidden Terminal Problem}

The hidden terminal problem is one of the most important challenges of the wireless communication to be handled carefully. As shown in Fig. 1, say host A starts a transmission to node $\mathrm{B}$. Meanwhile, host $\mathrm{C}$ also intends to start communication with host $B$. Since host $C$ is out of the transmission range of host $A$, is unaware of the ongoing transmission. Signals of both host A and $\mathrm{C}$ will collide at host B unwittingly which is called the hidden terminal problem [15$16]$.

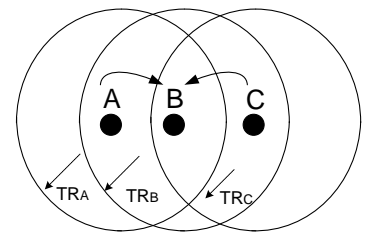

Fig. 1. An illustration of the hidden terminal problem

In the above-mentioned scenario as depicted in Fig. 1, it is not possible to prevent a possible collision by means of a Physical Carrier Sensing (PCS) mechanism. Thus, an alternative approach primarily proposed to prevent the hidden terminal is the MACA protocol which employs Virtual Carrier Sensing (VCS) mechanism rather than PCS. In this mechanism, the sender firstly sends a RTS control message including the amount of data it intends to send. Neighbors of the sender refrains themselves long enough from attempting to access the common transmission medium in order the CTS control message sent by the receiver to be acquired without any distortion at the sender side.

Meanwhile, when the receiver gets the RTS message, replies back with a CTS message also including the information about the duration of the communication. Neighbors that are in the vicinity of the receiver overhear the CTS message and defer their (if) intended transmissions during the data transmission period that will take place. As discussed in [9], many followers, amendments and new alternatives to the MACA have been proposed in the literature to date. However, the primary standard 802.11 is derived from the MACA protocol [17].

Depending on their receivers, message types in Local Area Networks (LANs) can be roughly split into two main categories:

- $\quad$ Broadcast messages that are not targeted to a specific receiver, i.e. every node in the vicinity of the sender are desired to get the packet. 
- Receiver specific messages that are destined to a specific host in the vicinity of the sender.

In infrastructure types of LANs, since there is a central coordinator, (i.e. access point), the broadcast packet generated by the sender is sent firstly to the access point by utilizing RTS/CTS mechanism. Access point then broadcasts it through the network. Unicast packets are also sent in the same way from the sender to the access point.

In ad-hoc type LANs, however, the mechanism is different for broadcast and unicast messages. As is known, for broadcast messages, there is no need to identify a receiver id in the packet. However, for the unicast packets, the receiver address should be included. Since there is not any central coordinator in an ad-hoc network, unicast packets are directly destined to the receiver host by a preceding RTS/CTS message exchange, whereas broadcast messages are directly pumped into the medium without any processor message exchange. That is because, for a broadcast message, it is not definite for the sender that how many CTS messages should be retrieved in order to start the data transmission. Thus, in ad-hoc networks, no RTS/CTS type control mechanism is applied during broadcast message transmission [18].

\section{B. Spatial Reusing \& Exposed Terminal Problem}

Another drawback of the wireless communication is the limited bandwidth resource. Since the frequency interval utilized for WLANs have become short of the capacity for the excessing demands of the users, researchers have been pursuing for the new solutions to mitigate this capacity shortage problem. The endeavor performed about capacity facilitation has been done at different layers of the protocol stack such as new data compression methods at the application layer, modulation techniques at the physical layer and MAC protocols at the data link layer.

The network capacity is highly related with the spatial reusing which can be described as a measure of the degree of the reuse of the spectrum per space or also can be expressed as the number of concurrent transmissions that can occur in the network without interfering each other [19-20]. By increasing the number of simultaneous transmissions that proceed in the network by means of spatial separation improves spatial reuse which inherently increases the overall network throughput. To achieve spatial reuse maximization, MAC protocol must coordinate the hosts to access the common medium depending on their distances to avoid interfering another ongoing transmission whilst not to prevent an upcoming nondestructive transmission but declared as possibly interferer because of the traditional PCS and VCS mechanisms [21].

4-phase MAC protocol of 802.11 alleviates highly the hidden terminal problem, whilst posing another challenge called exposed terminal problem which is a critical issue to be considered in order to improve spatial reuse [22]. As depicted in Fig. 2, although a transmission from node $\mathrm{C}$ to node $\mathrm{D}$ does not affect a transmission from node $B$ to $A$; node $C$ defers its transmission to node $\mathrm{D}$ unnecessarily because of the overheard RTS message sent by node $B$. A variety of research activities (e.g. [23-24]) have been performed to alleviate the exposed terminal problem thereby to increase the spatial reusing in the network which inherently results with the overall network throughput. An alternative solution offered by the community is the power control during transmissions that is discussed in detail in the following section.

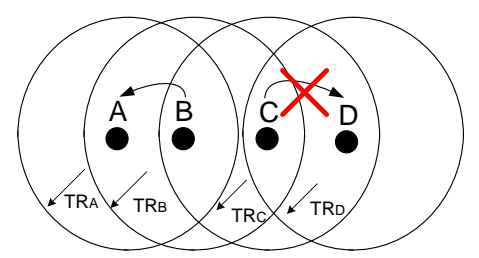

Fig. 2. An illustration of the exposed terminal problem

\section{Power Control}

As mentioned previously, limited capacity and power supply of the mobile devices are the primary drawbacks of wireless networks. Methods are being considered to improve spatial reuse and energy conservation for the sake of capacity and energy prudence. Power control is one of the choices offered for this purpose. Power saving can be achieved in two ways:

- Changing states of the devices to low-power mode during idle periods which the 802.11 Power Saving Management (PSM) is based on. Research studies to date have revealed that the highest energy consumption is attributed to the data communication unit [25-26]. However, if the hosts stay in the idle state longer times when compared with the data transmission period, it is not the best way purely striving to save power by transmission power control [27]. It is identified that a significant amount of energy is consumed by the devices at idle states even though they do not make any transmission [28]. Therefore, it is deduced that it is better for devices to change their states to passive position at which they require minimum energy rather than holding at a constant level [29].

- Original IEEE 802.11 applies constant power level during transmissions that is one of the primary prohibitive factors for the spatial reuse. Beyond the exposed terminal problem, it is sometimes possible for two transmissions to take place simultaneously without distorting each other by means of power control despite the RTS/CTS mechanism as illustrated in Fig. 3.

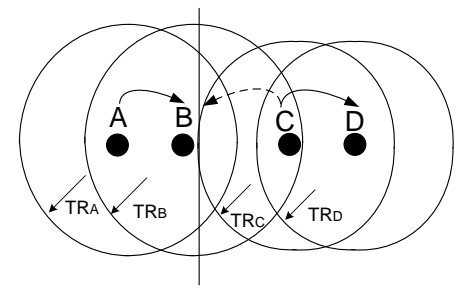

Fig. 3. An illustration of the power controlled data transmission

As depicted in Fig. 3, during a transmission between nodes A-B, a concurrent transmission can also take place between C$\mathrm{D}$, if node $\mathrm{C}$ can arrange its transmission power to the level that signals arriving to node $\mathrm{B}$ will not collide with the ones generated by node $\mathrm{A}$. 


\section{POWER CONTROLLED TRANSMISSION CHALLENGES AND SOLUTIONS}

As stated in the previous section, power controlled transmission can yield to significant energy savings as well as spatial reuse improvements that ultimately results with overall throughput enhancement. However, new challenges emerge with the power-controlled transmission, such as Hidden Terminal Jamming and Power Control Induced Hidden Terminal Problems. Following sections introduce these crucial issues that can be handled by different methods which will be discussed subsequently.

\section{A. Hidden Terminal Jamming Problem}

One of the challenges emerge by the power-controlled transmission is the Hidden Terminal Jamming that occurs during two concurrent transmissions of which different power levels employed for each.

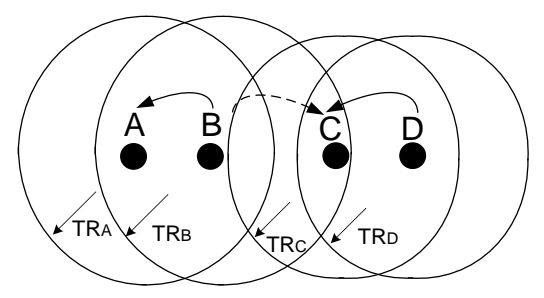

Fig. 4. Hidden Terminal Jamming Problem

In the scenario depicted in Fig. 4, say node $\mathrm{C}$ starts a transmission to node D. Meanwhile, node B starts a new transmission which is destined for node A. It is assumed that, all the control messages (RTS/CTS) and data packets are exchanged with the same power level. That is, if node sends a RTS message with a power level $\mathrm{Pt}$, it continues to send data packet with the same power level. Since this power level is lower enough that none of the RTS/CTS messages of node C arrive at node B; B is unaware of the ongoing transmission and starts to send its signals with a power level higher that covers also node $\mathrm{C}$. Therefore, signals sent by node $\mathrm{D}$ collide with the signals sent by node $\mathrm{B}$, which is called the Hidden Terminal Jamming Problem [27, 30]. An idea offered for mitigating the Hidden Terminal Jamming Problem is that, instead of using the same power level also for control messages, a higher power can be applied for these packets to make it possible to transmit them to every neighbor in the vicinity to notify them about the upcoming transmission. That is, RTS/CTS control message transmission should be performed at the maximum power level possible, but DATA/ACK packets can be transmitted at a minimum power level related with the distance [27, 31-32] as shown in Fig. 5.

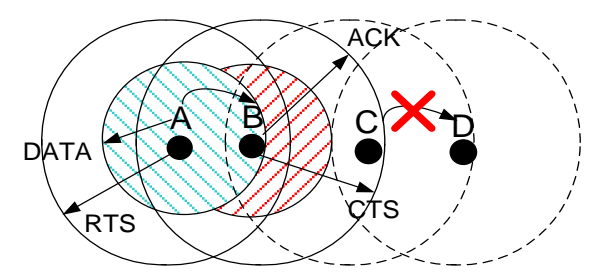

Fig. 5. Power level differentiation to mitigate the hidden terminal jamming problem

\section{B. Spatial reuse performance analysis of 802.11 RTS/CT}

Ye et.al [35] analysis the spatial reuse performance of IEE 802.11 provided that all the hosts in the network use the maximum power level during transmissions. In order to understand the challenges fairly, some definitions are introduced below [33-35]:

Transmission Range(RT): is the range within that a packet transmission is performed successfully and retrieved by the receiver correctly under the assumption that any other radio interference occurs.

Interference Range(RI): is the range within that the receiver host can be interfered by a sender(s) of another irrelevant transmission(s).

Most of the research studies [36-38] base on the two-way ground reflection model [39-40] as follows:

$$
P_{r}(d)=\frac{P_{t} G_{t} G_{r} h_{t}^{2} h_{r}^{2}}{d^{n} L}
$$

where $P_{r}(d)$ is the signal power at the receiver, $P_{t}$ denotes the transmitter power, $G_{t}$ and $G_{r}$ express the antenna gains respectively, $h_{t}$ and $h_{r}$ are the antenna heights, $d$ is the distance, and $L$ is the system loss. $n$ is the path loss exponent ranging from 2 to 6 depending on the pattern and environment that the communication takes place. A list of well accepted values for different types of environments is given in Table 1:

TABLE I. VALUES OF THE PATH LOSS EXPONENT $N$ FOR DifFERENT ENVIRONMENTS

\begin{tabular}{|l|l|}
\hline Environment & $\boldsymbol{n}$ \\
\hline Free space & 2 \\
Urban area & $2.7-3.5$ \\
Shadowed urban area & $3-5$ \\
Indoor & $4-6$ \\
\hline
\end{tabular}

By omitting the other parameters as done in almost all previous work and only considering the distance between the communicating pairs, Equation (1) takes the form:

$$
P_{r}(d)=P_{t} d^{-n}
$$

The relationship between the received (Pr) and interfering power levels at the receiver $(\mathrm{Pi})$ is given as follows:

$$
S I R=\frac{P_{r}}{P_{i}}=\left(\frac{R_{i}}{d}\right)^{n} \geq S I R_{T h r}
$$

where SIR denotes the signal-to-interference ratio (ambient noise is neglected) and SIRThr is the threshold capture value that is the ratio of the essence signal to the interfering signal arrived from another irrelevant transmitter. SIRThr is the reference value that the SIR value should be above in order the retrieved signal to be justified as meaningful and valuable. In their study, the values assigned to $S I R_{T h r}$ and $n$ are 10 and 4 respectively; say that the indoor environment is considered. When the abovementioned values are put properly, equation (3) takes the form:

$$
\begin{gathered}
S I R=\left(\frac{R_{i}}{d}\right)^{4}=10 \\
R_{i}=10^{1 / 4} d \cong 1,78 d
\end{gathered}
$$

As clarified in equation (5), the interference range (Ri) only depends on the distance between the sender and receiver; i.e. there is not any relationship between $\mathrm{Ri}$ and $\mathrm{Rt}$. 
Regarding to their comments, when the maximum transmission power level is utilized permanently, three situations emerge that should be considered separately:

- Underactive Scenario: In this scenario, the value of $d$ lays at the interval: $\left(R_{t} / 1,78\right) \leq d<R_{t}$. They express that in ns-2 and WaveLAN, $R_{t}$ is determined as $250 \mathrm{~m}$ for the maximum transmission power value of $P_{t}$. Thus, the values of the interval becomes as : $\left(0,56 R_{t}\right) \leq d<R_{t}=141 \leq d<250$

$R_{i}$ is larger than $R_{t}$ in this scenario as illustrated in Fig. 6:

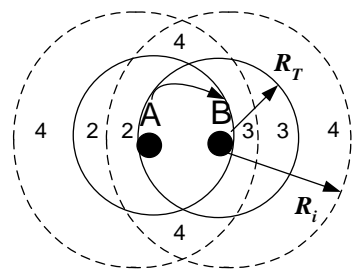

Fig. 6. $0,56 * R_{t} \leq d<R_{t}$.

A potential collision will be induced by the hosts deployed in the regions denoted by 4 , since these nodes are out of the transmission range $\left(R_{t}\right)$. Thus, they are unaware of the ongoing transmission and might start to send their own signals destined to a different receiver which will collide with the former.

- Moderate Scenario: $R_{i} \leq R_{t}$ in this scenario, of which $d$ lays at the interval $\left(R_{t} / 2,78\right) \leq d<\left(R_{t} / 1,78\right)=90 \leq d<$ 141

in Lucent's WaveLAN and ns-2.

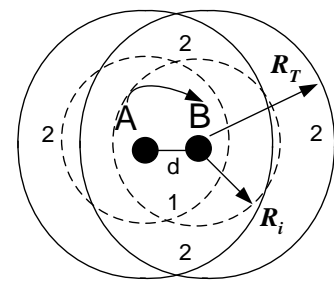

Fig. 7. $\left(R_{t} / 2,78\right) \leq d<\left(R_{t} / 1,78\right)$.

Despite the hosts deployed in the regions denoted by 2 do not interfere they will defer their transmissions regarding the reception of RTS/CTS control messages. Thus, 802.11 RTS/CTS VCS mechanism gives erroneous alarm and confines the throughput of the network.

- Overactive Scenario: $\mathrm{Ri}<\mathrm{Rt}$ in this scenario which models the real-life office, building or house LANs with approximate range of 100 m coverage. $d<\left(R_{t} / 2,78\right)=d<90$.

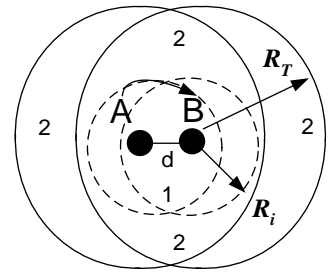

Fig. 8. $d<\left(R_{t} / 2,78\right)$.
As depicted in Fig. 8, though it is possible for concurrent transmissions to take place; unproductive region size increases. Hosts deployed in the regions expressed by 2 are not allowed to transmit because of the prohibitive behavior of 802.11 RTS/CTS VCS mechanism.

In essence, Scenario 2-3 concludes with the same result which is a reduction of the spatial reuse. In Scenario 1, the hidden terminal problem emerges this time. These scenarios clarify the deficiency of 802.11 RTS/CTS mechanism for the hidden terminal and spatial reuse issues. Besides the performance analysis of 802.11 RTS/CTS mechanism for spatial reusing, Ye et.al [35] propose a method to achieve improvement for the overactive case. That is, hosts deployed in region 2 in Fig. 7 that hear either a RTS or CTS but not both, can start their transmissions because they will not affect the ongoing transmission that take place between A-B.

\section{Power Control Induced Hidden Terminal Problem}

Significant improvements in energy saving are intended by means of power-controlled data transmission; especially for the type of networks in which data transmission periods takes considerably comparable with the idle state intervals in contrast to the wireless sensor network. Moreover, by decreasing the power of the transmitted signals, the range of the area that will be interfered shrinks as well. Hence, a possible concurrent communication in vicinity can take place without any distortion. However, the methods suggested previously such as utilizing different power levels for control and data packets can induce a new problem that is called as the Power Control Induced Hidden Terminal Problem (POINT) [33].

In the previous section, it is identified that, since the maximum transmission power is used permanently by all of the hosts in the network, $R_{i}$ does not depend on the transmitting power $\left(P_{t}\right)$. Shih et.al [33] discuss that, in order for a transmission to be valid, two conditions given below should be satisfied:

- The strength of the received signal should be greater than a threshold value: $P_{r} \geq P_{T h r}$.

- The ratio of the received signal strength to the total interfering signals strength that is the SIR should be also greater than the $S I R_{T h r}$.

$$
\begin{gathered}
S I R=\frac{P_{r}}{P_{i}} \geq S I R_{T h r} \\
P_{\alpha}=\max \left(P_{T h r}, P_{i} * S I R_{T h r}\right)
\end{gathered}
$$

In order to satisfy the abovementioned conditions, $P_{r} \geq P_{\alpha}$. Thus, basis on equation (2), transmitting range can be calculated as follows:

$$
R_{t}=\left(\frac{P_{t}}{P_{\alpha}}\right)^{1 / n}
$$

Since the transmit power levels of the hosts in the network are not fixed and identical, $R_{i}$ depends upon $P_{t}$ as well as the remaining tolerable interference level of the receiver which is calculated as follows:

$$
P_{i}=P_{c i}+P_{r i}
$$

where $P_{c i}, P_{r i}$ indicate the current strength of the total interfering signals and remaining tolerable interfering signal strength at the receiver. To satisfy the condition expressed in equation (6): 


$$
P_{r i} \leq \frac{P_{r}}{S I R_{T h r}}-P_{c i}
$$

In the vicinity of the receiver, say the closest node (with the distance $d_{X R}$ to the indicated receiver) denoted by $X$ starts a transmission with the maximum transmitting power $\left(P_{\max }\right)$. The signals emerged by that node will be sufficient to result a collision and corrupt the transmission at the receiver. Thus, $R_{i}$ can be calculated by considering only the signals originate from node $X$. As a result, equation (10) can be rewritten as follows:

$$
\frac{P_{\max }}{\left(d_{X R}\right)^{n}} \leq \frac{P_{r}}{S I R_{T h r}}-P_{c i}
$$

Thus, the distance $d_{X R}$ actually identifies the minimum range that a neighbor should be in proximity to start a noninterfering transmission with the worst case of using the maximum transmission $P_{\max }$. By this way, $R_{i}$ which in essence substitutes for $d_{X R}$ should satisfy the following condition:

$$
R_{i}\left(P_{t}\right)=\left(\frac{P_{\max }}{\left(\frac{P_{t}}{\left(d_{X R}\right)^{n^{* S I R} T h r}}\right)-P_{c i}}\right)^{1 / n}
$$

In the following section, the description of the POINT problem is identified and how it can be mitigated by arranging the transmission power inferred from the equations introduced thus far. Assume a scenario as illustrated in Fig. 9, in which host A sends RTS control message with $P_{\max }$ and host B replies back with CTS message with $P_{\max }$ also.

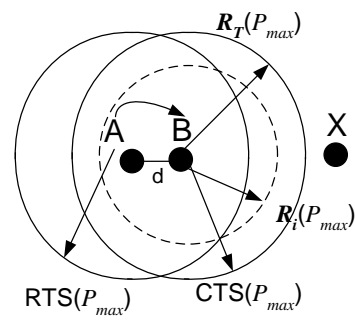

(a)

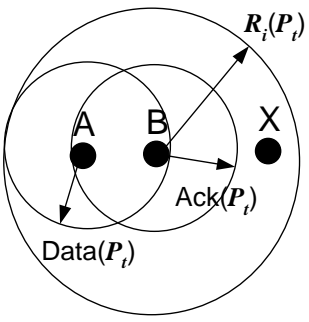

(b)
Fig. 9. A sample scenario to illustrate the POINT problem

$\mathrm{X}$ is unaware of the ongoing transmission since none of the RTS/CTS packets arrive it. As depicted in Fig. 9(a), X is obviously out of the interfering range and can start its own transmission. However, when host $\mathrm{A}$ attempts to make a transmission with a power level $P_{t}$ that is lower than $P_{\max }$, host $\mathrm{X}$ which was formerly out of $R_{i}$ involves in the interference range. The reason is that the interference range $\left(R_{i}\right)$ extends by the reduction in the transmission power $\left(P_{t}\right)$.

Shih et.al [33] propose the Collision Avoidance Power Control (CPAC) MAC protocol to mitigate the POINT problem that emerges especially in the situations that $d<$ $\left(R_{t} / 2,78\right)$. In CPAC, instead of using the exact power level related with the distance, utilizing an appropriate power level will prevent unexpected interferers to emerge. The aforementioned appropriate power level $P_{a p p}$ is calculated as follows. Since $R_{i}$ and $R_{t}\left(P_{\text {max }}\right)$ are intended to be equal in CPAC, $P_{\text {app }}$ can be derived by considering equation (8) and (12) as follows:

$$
\begin{gathered}
R_{t}\left(P_{\text {max }}\right)=\left(\frac{P_{\max }}{P_{\alpha}}\right)^{1 / n}=\left(\frac{P_{\max }}{\left(\frac{P_{a p p}}{\left(d_{X R}\right)^{n_{*} S I R} T h r}\right)-P_{c i}}\right)^{1 / n}=R_{i}\left(P_{\text {max }}\right) \\
P_{\text {app }} \text { is derived as follows: } \\
P_{\text {app }}=\left(P_{\alpha}+P_{c i}\right) d^{n} S I R_{T h r}
\end{gathered}
$$

Hence, POINT problem can be alleviated by arranging the applied transmitting power level as discussed in CAPC [33].

\section{CONCLUSIONS}

The throughput and delay performance of WANETs highly depends on their ability of spatial reusing. Power control is a way of improving spatial reuse, as well as a promising solution for energy conservation. 802.11 RTS/CTS has some drawbacks from the point of view of spatial reuse and power efficiency. By applying varying transmission power level, it is desired to improve the spatial reusing. This time, new problems such as Hidden Terminal Jamming and POINT emerge that should be exhaustively considered. In this article, we discuss the primary details about the power usage and throughput deficiency of the traditional 802.11 RTS/CTS mechanism. Improvements by means of power control are introduced as well as the solutions to the challenges likely to emerge because of the usage of diverse power levels throughout the network.

\section{REFERENCES}

[1] S. Umamaheswaran, N. Kumar, and P.K. Biswas, “A Survey of Routing Protocol Design Patterns for Mobile Ad Hoc Networks”, In Proc. of the $2^{\text {nd }}$ International Conference on Emerging Technology Trends in Electronics, Communication and Networking, pp. 1-6, 2014.

[2] M. Natkaniec, K. K. Szott, S. Szott, and G. Bianchi, "A Survey of Medium Access Mechanisms for Providing QoS in Ad-Hoc Networks”, IEEE Communications Surveys \& Tutorials, vol. 15, No. 2, pp. 592620, 2013.

[3] L. Shengbin, and Z. Xiaoliang, “A Survey on Mac Layer in Ad Hoc Wireless Networks”, Communications in Computer and Information Science, vol. 225, pp. 691-699, 2011.

[4] M. Conti, and S. Giordano, "Mobile Ad Hoc Networking: Milestones, Challenges, and New Research Directions”, Ad Hoc and Sensor Networks, vol. 52, no. 1, pp. 85-96, 2014.

[5] M. Conti, and S. Giordano, "Multihop Ad Hoc Networking: The Theory”, IEEE Communications Magazine, vol. 45, no. 4, pp. 78-86, 2007.

[6] G. Anastasi, M. Conti, and A. Passarella, "Power Management in Mobile and Pervasive Computing Systems," Ch. 24, Algorithms and Protocols for Wireless and Mobile Networks, A. Boukerche, Ed., CRCHall, Oct. 2005.

[7] A. Ali, W. Huiqiangi, L. Hongwu, and X. Chen, "A Survey of MAC Protocols Design Strategies and Techniques in Wireless Ad Hoc Networks”, Journal of Communications, vol. 9, no. 1, pp. 30-38, 2014.

[8] L. Kleinrock, and F. Tobagi, "Packet Switching in Radio Channels, part II - the Hidden Terminal Problem in Carrier Sense Multiple Access and the Busy Tone Solution”, IEEE Transactions on Communications, vol. 23, no. 12, pp. 1417-1433, 1975.

[9] K. K. Szott, “A Survvey of MAC Layer Solutions to the Hidden Node Problem in Ad-hoc Networks”, Ad Hoc Networks, vol. 10, pp. 635-660, 2012.

[10] P. Karn, "MACA - a New Channel Access Method for Packet Radio", In Proceedings of the ARRL/CRRL Amateur Radio $9^{\text {th }}$ Computer Networking Conference, vol. 9. pp. 134-140, 1990.

[11] Wireless LAN Medium Access Control (MAC) and Physical Layer (PHY) Specifications, IEEE STd. 802.11-1999, 1999.

[12] J. Xu, Y. Jiang, and A. Perkis, "Towards Analysis of Intra-Flow Contention in Multi-hop Wireless Networks", In Proc. of the $6^{\text {th }}$ International Conference on Mobile Ad-hoc and Sensor Networks (MSN), pp.176-184, 2010. 
[13] U. Roedig, A. Barroso, and C. J. Sreenan, "f-MAC: A Deterministic Media Access Control Protocol without Time Synchronization”, In Proc. of the $3^{\text {rd }}$ European Workshop on Wireless Sensor Network, pp. 276291, 2006.

[14] M. Ghazvini, N. Movahedinia, and K. Jamshidi, “A Game Theory based Contention Window Adjustment for IEEE 802.11 under Heavy Load", International Journal of Communication Networks and Information Security, vol. 5, no. 2, pp. 93-103, 2013.

[15] Y. Neeraja, V. Sumalatha, and M. Begum, "Comprehensive Survey of Medium Access Control Protocols for MANETs", International Journal of Emerging Trends \& Technology in Computer Science, vol. 2, no. 3, pp. 211-219, 2013.

[16] S. Kumar, V. S. Raghavan, and J. Deng, "Medium Access Control Protocols for Ad Hoc Wireless Networks: A Survey", Ad Hoc Networks, vol. 4, pp. 326-358, 2006.

[17] N. Poojary, S. V. Krishnamurthy, and S. Dao, "Medium Access Control in a Network of Ad Hoc Mobile Nodes with Heterogeneous Power Capabilities", In Proc. of the IEEE International Conference on Communications (ICC 2001), vol. 3, pp. 872-877, 2001.

[18] S. Agarwal, R. H. Katz, and S. V. Krishnamurthy, and S. K. Dao, "Distributed Power Control in Ad-hoc Wireless Networks", In Proc. of the $12^{\text {th }}$ IEEE International Symposium on Personal, Indoor and Mobile Radio Communications, vol. 2, pp. 59-66, 2001.

[19] Y. Kim, F. Baccelli, G. Veciana, "Spatial Reuse and Fairness of Ad Hoc Networks with Channel-Aware CSMA Protocols", IEEE Transaction on Information Theory, vol. 60, no. 7, pp. 4139-4157, 2014.

[20] T. S. Kim, H. Lim, J. C. Hou, "Understanding and Improving the Spatial Reuse in Multihop Wireless Networks”, vol. 7, no. 10, pp. 1200-1212, 2008.

[21] J. Zhu, X. Guo, L. L. Yang, W. S. Conner, S. Roy, and M. M. Hazra, "Adapting physical carrier sensing to maximize spatial reuse in 802.11 mesh networks", Wireless Communications and Mobile Computing, vol. 4, no. 8, pp. 933-946, 2004.

[22] N. Santhapuri, S. Nelakuditi, and R. R. Choudhury, "On Spatial Reuse and Capture in Ad Hoc Networks", In proc. of the IEEE Wireless Communications and Networking Conference (WCNC 2008), pp. 16281633, 2008.

[23] A. Acharya, A. Misra, and S. Bansal, "MACA-P: A MAC for Concurrent Transmissions in Multi-hop Wireless Networks", In Proc. Of the $1^{\text {st }}$ IEEE International Conference on Pervasive Computing and Communications (PerCom 2003), pp. 505-509, 2003.

[24] N. Santhpuri, J. Wang, Z. Zhong, S. Nelakuditi, "Piggybacked-Ackaided Concurrent Transmissions", in ICNP Poster Session, 2005.

[25] J. P. Ebert, B. Stremmel, E. Wiederhold, and A. Wolisz, "An Energyefficient Power Control Approach for WLANs", Journal Of Communications and Networks, vol.2, no.3, pp. 197-206, 2000.

[26] L. M. Feeney, and M. Nilsson, "Investigating the Energy Consumption of a Wireless Network Interface in an Ad Hoc Networking Environment”, In Proc. of the $20^{\text {th }}$ Annual Joint Conference of the IEEE Computer and Communications Societies, vol. 3, pp. 1548-1557, 2001.
[27] S. Gobriel, and R. Melhem, Daniel Mossé, “A Unified Interference/Collision Model for Optimal MAC Transmission Power in Adhoc Networks", International Journal of Wireless and Mobile Computing, vol.1, no.3/4, pp. 179 - 190, 2006.

[28] R. Zheng, J. C. Hou, and L. Sha, "Power management and power control in wireless networks", Frontiers in Artificial Intelligence and Applications, vol. 14, no. 1, pp. 1-25, 2007.

[29] R. Zheng, J. C. Hou, and L. Sha, "Performance analysis of the IEEE 802.11 power saving mode",

https://www.researchgate.net/publication/228751628_Performance_analysis_ of_the_IEEE_802.11_power_saving_mode.

[30] C. Ware, T. Wysocki, and J. Chicharo, "Hidden Terminal Jamming Problems in IEEE 802.11 Mobile Ad Hoc Networks", In Proc. of the IEEE International Conference on Communications, vol. 1, pp. 261-265, 2001.

[31] J. Gomez, A. T. Campbell, M. Naghshineh, and C. Bisdikian, "Conserving Transmission Power in Wireless Ad Hoc Networks", In Proc. of the $9^{\text {th }}$ International Conference on Network Protocols, pp. 2434, 2001.

[32] M. B. Pursley, H. B. Russell, and J. S. Wysocarski, "Energy-Efficient Transmission and Routing Protocols for Wireless Multiple-Hop Networks and Spread-Spectrum Radios”, In Proc. of the International Conference on Information Systems for Enhanced Public Safety and Security (EUROCOMM 2000), pp. 1-5, 2000.

[33] K. P. Shih, and Y. D. Chen, "CAPC: A Collision Avoidance Power Control MAC Protocol for Wireless Ad Hoc Networks", IEEE Communications Letters, vol. 9, no. 9, pp. 869-861, 2005.

[34] K. Xu, M. Gerla, and S. Bae, "How Effective is the IEEE 802.11 RTSKTS Handshake in Ad Hoc Networks", In Proc. of the IEEE Global Telecommunications Conference (GLOBECOM '02), vol. 1, pp. 72-76, 2002.

[35] F. Ye, and S. Yi, Biplab Sikdar, Improving Spatial Reuse of IEEE 802.11 Based Ad Hoc Networks", In Proc. of the IEEE Global Telecommunications Conference (GLOBECOM '03), vol. 2, pp. 10131017, 2003.

[36] R. Hekmat, and P. V. Mieghem, "Interference in Wireless Multi-hop Ad-hoc Networks and its Effect on Network Capacity”, Wireless Networks, vol. 10, no 4, pp. 389-399, 2004.

[37] J. Zhu, X. Guo, L. L. Yang, and W. S. Conner, "Leveraging Spatial Reuse in 802.11 Mesh Networks with Enhanced Physical Carrier Sensing”, In Proc. of the IEEE International Conference on Communications, vol. 7, pp. 4004-4011, 2004.

[38] D. N. M. Dang, C. S. Hong, S. Lee, and J. Lee, "A SINR-Based MAC Protocol for Wireless Ad Hoc Networks", IEEE Communications Letters, vol. 16, no. 2, pp. 2016-2019, 2012.

[39] T. S. Rappaport, "Wireless Communications: Principles and Practice", Prentice Hall, New Jersey, 1996.

[40] R. Prasad, "Universal Wireless Personal Communications", Artech House, 1998. 